\title{
Prevalence of Anemia in Egypt (Al-Gharbia Governorate)
}

\author{
1* Ghada Z A Soliman; ${ }^{2 *}$ Magdi N Azmi, \& ${ }^{3 * *}$ Soha El-S. \\ ${ }^{1}$ Biochemistry; ${ }^{2}$ Nutrition Education \& Ecological Science $;{ }^{3}$ Nutrition. \\ *National Nutrition Institute, Cairo, Egypt; ** Suez Canal University, Suez, Egypt
}

\begin{abstract}
Introduction: Iron deficiency anemia is the most commen type of anemia related to mal nutrition world wide. It represents a major problem in developing countries, especially in Egypt.

Purpose: The present study was designed to assess the prevalence and status of anemia in Al-Gharbia Governorate in Egypt.

Material and Methods: 649 mothers and one of their sibling were chosen from different districts with different food habits and socio-economic status.Hb were determined in mothers and sibling. Anthropometric measurement includes measurements of height and weight was done. Age was recorded. Education, Job, income; marital status and number of personal/family are recorded when possible.

Results: No severe anemia $(\mathrm{Hb}<7 \mathrm{~g} / \mathrm{dl})$ was found in any studied group (mothers or sibling), only moderate to mild anemia was found $((\mathrm{Hb}>7 \mathrm{~g} / \mathrm{dl})$. The prevalence of anemia in mother's is $>47 \%$, which represent a problem of high degree, while, the prevalence of anemia in sibling is $52.25 \& 54.03 \%$ for male and female respectively which represent a problem of high degree. The cause of anemia in mothers may be due to menstrual blood loss, increased duration of menstrual blood flow, while in children may be due to anemic mother, poor, diet, bad food habit.
\end{abstract}

Conclusion: To reduce prevalence of anemia and improve anemic mothers, sibling status in Egypt we needs to improve iron supplementation program

Key Words: Egypt, Anemia, Women,

\section{Introduction}

The World Bank estimates that onehalf of the world's population suffers from malnutrition and that two million people suffer from diseases related to iron, iodine and vitamin A deficiency (World Bank 1996). These deficiencies affect women, infants and children especially those of developing countries. Poor eating habits play a major role in the development of iron deficiency anemia that is an important indicator of poor health status. Children and adolescent are at increased risk of developing iron deficiency anemia because of their increased demand for iron during growth and puppetry. In most cases, they are undiagnosed because of irregular, far visiting of heath clinics, doctors, and hospitals (World Bank 1996).

Iron-deficiency anemia (IDA) in infants is a common problem worldwide and an enormous public health risk in developing countries. The prevalence of anemia in infants varies between 5\% and $43 \%$ according to the population studied and the cut-off hemoglobin level used to define anemia in infancy (Florentino \& Guirriec 1984; deMeyer \& Adiels-Tegman 1985; UNAC $^{1}$ 1987; and Stolitzfus 2001 a \& b).

In developing countries, $40-45 \%$ of children aged 0-4 y old suffer from anemia (ACC/SCN 2000). The main cause of anemia is iron deficiency (ACC/SCN 2000). Severe anemia increases mortality; iron deficiency impairs behavioral and cognitive development and reduces fitness and work capacity (Stoltzfus $2001 \mathrm{a} \& \mathrm{~b}$ ).

Anemia is caused either by limited dietary intake, excessive loss of nutrients or excessive utilization. The anemia is associated with a number of sequelae including both structural chan-ges, like mitochondrial swelling and mucosal atrophy, and functional abnor-malities, such as cardiac failure, decre-ased work output,

\footnotetext{
${ }^{1}$ UNAC: United Nations Administrative Committee.
} 
increased pregnancy risks and increased susceptibility to infections

Globally, iron deficiency anemia ranks number 9 among 26 risk factors included in the Global Burden of Disease (GBD) 2000, and accounts for 841,000 deaths and 35,057,000 disability-adjusted life years lost. Africa and parts of Asia bear $71 \%$ of the global mortality burden and $65 \%$ of the disability-adjusted life years lost (WHO 2001, Stoltzfus 2003).

With $40 \%$ prevalence of anemia in the world, the prevalence in the developing countries tends to be three to four times higher than in the developed countries (Karkar and Kotecha 2004).

\section{Aim Of The Work}

The present study was designed to assess the prevalence and status of anemia in Al-Gharbia Governorate in Egypt.

\section{Material And Methods}

\section{Subjects}

The study was conducted in AlGharbia Governorate in Egypt during years 2003-2005. It includes 649 families of different ages, socioeconomic status with different food habits. The families included in the study were from different sites in the Governorate.

A social demographic profile including mothers' education, income of the family, age of both mother and one of her sibling, gender of the sibling, family structure and no of personal/family. General status of mothers and sibling were recorded.

\section{Biochemical Analysis}

Samples were collected for estimation of hemoglobin $(\mathrm{Hb})$ by cyanmethemoglobin method (Van Kampen and Zijlstra 1961).

\section{Anthropometic Data}

Anthropometric measurement includes measurements of height and weight according to standard WHO procedures (WHO Working Group, 1986) were done.

Weight was measured to the nearest $0.1 \mathrm{~kg}$ on a battery-powered digital balance and heights to the nearest $0.1 \mathrm{~cm}$ using a wooden length-measuring board with a sliding head bar (WHO Working Group, 1986) for individual $>2$ years. For children $<2$ years a specific wooden lengthmeasuring board and specific balance is used. The body mass index (BMI) was calculated as weight $/$ height $^{2}\left(\mathrm{~kg} / \mathrm{m}^{2}\right)$ and thinness defined as BMI for- age below the fifth percentile of the NHANES I reference population (WHO Expert Committee on Physical Status, 1995) or Z-scores below -2 SD. Height for age Z-scores was calculated using the sex specific $1978 \mathrm{CDC} / \mathrm{WHO}$ normalized version of the 1977 NCHS reference data in Epi Info2000 (Centers for Disease Control and Prevention, Atlanta, US). Stunting was defined as height-for-age Z-scores below -2 SD (WHO Expert Committee on Physical Status, 1995).

Age was recorded from the reported date of birth in school records, birthday certificate, where possible.

\section{Statistical Analysis}

Data are expressed as Mean \pm SE. Data were assessed by t-test (Avram 1964; and Steel \& Torrie 1969). Analysis was performed using Epi Info 2000 and SPSS 13. The correlations coefficients were assessed by Pearson's simple linear regression analysis (Avram 1964; and Steel \& Torrie 1969). P-values $<0.05$ were considered statistically significant.

\section{Criteria for Detecting Anemia}

The criteria for detecting anemia were diagnosed as WHO guidelines, (values less than $12 \mathrm{~g} / \mathrm{dL}$ for girls from 12 to 18 years and boys less than 14 years and less than 13 $\mathrm{g} / \mathrm{dL}$ for boys from 15 to 18 yrs of age, WHO/UNICEF/UNU 1996, 2001). Cut-off values have been established for groups of different ages and sex, starting from the age of 6 months (CDC 1998 criteria, WHO/ UNICEF/UNU ${ }^{2}$ 1997). The cut-off value for children aged 6 months to $5 \mathrm{y}$ is $11 \mathrm{~g} / \mathrm{dl}$ (Table 1).

\section{Degree of Anemia}

Mild anemia is diagnosed when $\mathrm{Hb}$ concentration is above $10.0 \mathrm{~g} / \mathrm{dl}$ but below the cut-off level, moderate anemia when the concentration between 7 and $<10.0 \mathrm{~g} / \mathrm{dl}$ and severe anemia when it is below $7 \mathrm{~g} / \mathrm{dl}$.

\footnotetext{
${ }^{2}$ UNU: United Nations University
} 
On a population level, anemia prevalence can be distinguished as low, medium or high according to the level of prevalence suggested by WHO 1996 (Table 2).

\section{Results}

The present study was conducted to investigate the prevalence of anemia on AlGharbia Governorate in Egypt. The study includes 649 family representing 649 women. Of the 649 mothers enrolled in the study, $27(28.5 \%)$ refused to do Hb determination, of the 622 siblings enrolled in the study, $189(30.39 \%)$ refused to do Hb determination. $27(4.16 \%)$ of the mothers didn't allow their children to be measured or analyze $\mathrm{Hb}$. The mean age of the mothers in the total sample is $32.10 \pm 0.27$ with a range: $17-\leq 60 \mathrm{y}$, while the mean age of the male sibling in the total sample is $6.93 \pm 0.30 \mathrm{y}$, and for female sibling is $7.27 \pm 0.34 \mathrm{y}$ with a range: $0.5-30$ y. Number of male sibling participating in the study is higher the female one (M: F, 355: 267, 57.1: 42.9\%).

Table (3) shows distribution of mothers according to age, income, job, marital status and number of personal/family. The income of around $75 \%$ of the family is $\leq 200.0$ LE. $85.7 \%$ of the mothers are jobless. $94.9 \%$ of the mothers are married. Most of the families are big where number of personal/family ranges 2 - 9 persons with an average of 5 .

Table (4) shows prevalence (\%) of anemia in the studied mothers in different physiological status. A significant increase was found between $\mathrm{Hb}$ of non-pregnant lactating (NP L) and pregnant lactating ( $\mathrm{P}$ L) and also between non-pregnant nonlactating (NP NL) and pregnant nonlactating ( $\mathrm{P}$ NL) of the non- anæmic mothers. Also a significant increase was found between $\mathrm{Hb}$ of non-pregnant lactating (NP L) and non-pregnant nonlactating (NP NL) of the mothers. Prevalence (\%) of anemia in the P L, NP L and NP NL of studied mothers is $\geq 47 \%$ which represent a high problem according to table 2, while prevalence of anemia in the whole anemic sample (200 mother) is $45.26 \%$ which represent a high problem.

Table (5) shows prevalence (\%) of anemia among mothers in different physiol- ogical states according to age group. Prevalence (\%) of anemia among mothers $\leq$ $20 \mathrm{y}$ is $37.04 \%$ which represent a medium problem while prevalence (\%) of anemia among mothers $\geq 20-\leq 60 \mathrm{y}$ is $>43.0 \%$ which represent a high problem. Table (5) also shows that $56.4 \%$ of the mothers are illiterate, $91.4 \%$ are not pregnant, and 80.6 $\%$ are non-lactating.

Table (6) shows distribution of the sibling according to sex, age, weight, height, BMI and $\mathrm{Hb}$. The number of male sibling participating in this study is higher than female sibling $(32.96 \%)$. No significant differences were found between age, weight and height of male and its respective female sibling (according to age group).

Table (7) shows prevalence of anemia in the studied sibling according to age group. The prevalence of anemia in preschool children (6-60 months) is 49.48 \& $56.12 \%$ for male and female respectively where female shows higher prevalence. In general, the prevalence of anemia in this study is $52.25 \& 54.03 \%$ for male and female respectively where female shows slightly higher prevalence rate. The high prevalence of anemia in male adolescent was noticed at age group 13-14 y where it reach $83.33 \%$ while the high prevalence of anemia in female sibling was noticed at age group $\geq 19$ y where it reach $100 \%$.

No significant correlation was found between anemic mothers and their children or between anemic children and their mothers, which disagree with Joseph et al. (2006).

In this study no severe anemia $(\mathrm{Hb}<$ $7 \mathrm{~g} / \mathrm{dl}$ ) was found in any studied group (mothers or sibling), only moderate to mild anemia was found $((\mathrm{Hb}>7 \mathrm{~g} / \mathrm{dl})$.

Table (8) shows Z-Score of height/age; weight/age; weight/height of the studied sibling. HAZ shows that $\geq 53 \%$ of the sibling (male $\&$ female) are within normal range while $\geq 10.2 \%$ are tall and $\geq$ $30.4 \%$ are stunted. WAZ shows that $\geq 75.2$ $\%$ of the sibling (male $\&$ female) are within normal range while $\geq 4 \%$ are over weight and $\geq 15.2 \%$ are underweight. WHZ shows that $\geq 77.1 \%$ of the sibling (male \& female) are within normal range while $\geq$ $11.6 \%$ over weight and $\geq 7.7 \%$ are wasting. 


\section{Prevalence of Anemia in Egypt (Al-Gharbia Governorate)}

Table (1): The cut-off value for anemia

\begin{tabular}{|c|c|c|c|c|c|c|c|c|}
\hline & \multicolumn{2}{|c|}{ Children } & Girls & Boys & Boys & $\begin{array}{c}\text { Non } \\
\text { pregnant }\end{array}$ & Pregnant \\
\hline Age & $\begin{array}{c}6-60 \\
\text { month }\end{array}$ & $\begin{array}{c}5- \\
11 \mathrm{y}\end{array}$ & $\begin{array}{c}12-13 \\
\mathrm{y}\end{array}$ & $\begin{array}{c}<12-18 \\
\mathrm{y}\end{array}$ & $\begin{array}{c}<14 \\
\mathrm{y}\end{array}$ & $\begin{array}{c}15-18 \\
\mathrm{y}\end{array}$ & & \\
\hline $\begin{array}{c}\mathrm{Hb} \\
(\mathrm{g} / \mathrm{dl})\end{array}$ & 11.0 & 11.5 & 12.0 & 12.0 & 12.0 & 13.0 & 12.0 & 11.0 \\
\hline
\end{tabular}

Table (2): Prevalence of anemia on a population level.

\begin{tabular}{|c|c|c|c|}
\hline Level & Low & Medium & High \\
\hline$\%$ Prevalence & $<15 \%$ & $15-40 \%$ & $>40 \%$ \\
\hline
\end{tabular}

Table (3): Distribution of mothers according to age, income, job, marital status and number of personal/family.

\begin{tabular}{|c|c|c|c|c|c|c|c|c|c|c|c|c|c|c|c|c|c|c|c|c|c|c|c|}
\hline & \multirow[t]{2}{*}{ Age } & \multirow[t]{2}{*}{ No } & \multirow{2}{*}{\begin{tabular}{|l} 
Age $(y)$ \\
$M \pm S E$ \\
\end{tabular}} & \multicolumn{6}{|l|}{$\begin{array}{l}\text { Income } \\
\text { (LE) }\end{array}$} & \multicolumn{2}{|l|}{ Job } & \multicolumn{3}{|c|}{\begin{tabular}{|l} 
Marital \\
Status
\end{tabular}} & \multicolumn{9}{|l|}{\begin{tabular}{|c|} 
No of \\
Person/hous \\
$\mathrm{e}$
\end{tabular}} \\
\hline & & & & $\mathrm{M} \pm \mathrm{SE}$ & \multicolumn{5}{|c|}{\begin{tabular}{|l|l|l|l|l|}
$\leq 100$ & $\leq 200$ & $\leq 300$ & $\leq 400$ & $\leq 500$ \\
\end{tabular}} & 1 & 2 & 1 & 2 & 3 & $\mathrm{M} \pm \mathrm{SE}$ & 2 & 3 & 4 & 5 & 6 & 7 & 8 & 9 \\
\hline & & & & & & & & & & & & & & & & & & & & & & & \\
\hline 1 & $\leq 20 \mathrm{Y}$ & 31 & $18.82 \pm 0.12$ & $190.16 \pm 16.28$ & 3.2 & 71.0 & 12.9 & 12.9 & & 6.5 & 90.3 & 93.5 & & & $4.74 \pm 0.23$ & & 16.1 & 35.5 & 19.4 & 16.1 & 12.9 & 3.2 & \\
\hline 2 & $\leq 30 \mathrm{Y}$ & 249 & $26.65 \pm 0.17$ & $181.65 \pm 3.25$ & 4.4 & 77.1 & 17.7 & 0.4 & 0.4 & 10.0 & 88.4 & 94.4 & 1.2 & 0.4 & $4.75 \pm 0.08$ & 0.4 & 14.0 & 31.7 & 28.1 & 16.9 & 3.6 & 4.0 & 0.4 \\
\hline 3 & $<10 \mathrm{y}$ & 307 & $352+015$ & 1 & 12 & $78 ?$ & 163 & 10 & $0=2$ & | 121 & 857 & 054 & 30 & & $480+008$ & 03 & $14]_{1}$ & 207 & 270 & 153 & | & $23_{1}$ & 07 \\
\hline & $I=40 \mathrm{Y}$ & | & 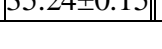 & $100.50 \pm 3.04$ & 4.2 & 10.2 & 10.2 & 1.0 & 0.3 & $\| 12.1$ & $\mid$ & 95.4 & P.9 & & $4.09 \pm 0.00$ & 0.5 & 14.5 & 20.1 & 21.0 & $\mid 15.3$ & 111.4 & & 0.1 \\
\hline 4 & $\leq 50 \mathrm{Y}$ & 52 & $43.56 \pm 0.22$ & $200.67 \pm 13.29$ & 7.69 & $\begin{array}{c}57.6 \\
9\end{array}$ & $\begin{array}{c}28.8 \\
5\end{array}$ & 1.92 & 3.85 & 7 & 41 & 49 & & & $5.10 \pm 0.18$ & & 7.69 & 21.15 & 22 & $\begin{array}{c}42.3 \\
1\end{array}$ & 3.85 & 3.851 & 1.92 \\
\hline & & & & & & & & & & & & & & & & & & & & & & & \\
\hline 5 & $\leq 60 \mathrm{Y}$ & 10 & $52.60 \pm 0.45$ & $195.40 \pm 16.32$ & & 70.0 & 30.0 & & & |50.0 & 40.0 & 100 & & & $4.70 \pm 0.21$ & & & 40.0 & 50.0 & 10.0 & & & \\
\hline & Total & 649 & $32.10 \pm 0.27$ & $183.18 \pm 2.36$ & 4.5 & 75.7 & 17.9 & 19 & 0.6 & $\mid 11.7$ & 85.7 & 94.9 & 0.8 & 0.2 & $4.84+0.05$ & 0.3 & $\mid 13.9$ & 297 & 28.7 & 16.2 & 77 & 29 & 0.6 \\
\hline
\end{tabular}

Job: 1: Working, 2: not working; Marital Status: 1: Married, 2: Widow, 3: Divorced

Table (4): Prevalence (\%) of anemia in the studied mothers in different physiological status

\begin{tabular}{|c|c|c|c|c|c|c|c|c|c|c|c|c|c|c|}
\hline & & \multicolumn{6}{|c|}{ Anemia } & \multicolumn{5}{|l|}{$\mathrm{Hb}(\mathrm{g} / \mathrm{dl})$} & \multirow{3}{*}{\multicolumn{2}{|c|}{ Total }} \\
\hline & & & & & & & & & & 3 & & & & \\
\hline & & & \multicolumn{2}{|l|}{$\mathrm{b}$} & Moderate & \multicolumn{2}{|l|}{$\mathrm{c}$} & \multicolumn{2}{|l|}{ Mild } & \multicolumn{3}{|r|}{ Non Anemic } & & \\
\hline & & No & No & $\%$ & $\mathrm{M} \pm \mathrm{SE}$ & No & $\%$ & $\mathrm{M} \pm \mathrm{SE}$ & & No & $\%$ & $\mathrm{M} \pm \mathrm{SE}$ & No & $\mathrm{M} \pm \mathrm{SE}$ \\
\hline \multirow[t]{2}{*}{ Pregnant } & Lactating & 7 & 2 & 33.33 & $9.93 \pm 0.43$ & 1 & 16.67 & 10.8 & & 3 & 50.0 & $12.03 \pm 0.37$ & 6 & $10.98 \pm 0.49$ \\
\hline & Non Lactating & 49 & 3 & 8.57 & $9.43 \pm 0.23$ & 3 & 8.57 & $10.93 \pm 0.07$ & & 298 & 82.86 & $12.18 \pm 0.14$ & 35 & $11.83 \pm 0.18$ \\
\hline \multirow[t]{2}{*}{ Non Pregnant } & Lactating & 119 & 17 & 20.73 & $9.18 \pm 0.17$ & 24 & 29.27 & $11.12 \pm 0.10$ & & 41 & 50 & $13.21 \pm 0.12$ & 82 & $11.76 \pm 0.19$ \\
\hline & Non Lactating & 474 & 25 & 7.33 & $9.46 \pm 0.07$ & 135 & 39.59 & $11.34 \pm 0.04$ & & 1815 & 53.08 & $13.11 \pm 0.06$ & 341 & $12.14 \pm 0.07$ \\
\hline Total & & 649 & 47 & & & 163 & & & & 254 & & & 464 & \\
\hline$\%$ Anemic & & & & & & & & & 45.26 & & & & & \\
\hline
\end{tabular}


Ghada Z A Soliman et al

Table (5): prevalence of anemia among mothers in different physiological states according to age group

\begin{tabular}{|c|c|c|c|c|c|c|c|c|c|c|c|c|c|c|c|c|c|c|}
\hline \multirow[t]{2}{*}{$\begin{array}{l}\Omega \\
0 \\
\vdots \\
\vdots \\
z \\
0\end{array}$} & & \multirow[t]{2}{*}{ No } & \multicolumn{5}{|c|}{ 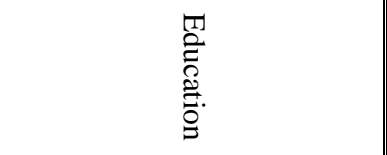 } & \multicolumn{3}{|l|}{ 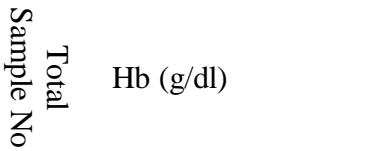 } & \multirow[t]{2}{*}{ 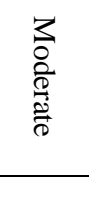 } & \multirow[t]{2}{*}{3} & \multirow[t]{2}{*}{ 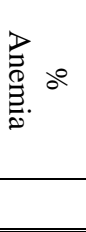 } & \multirow[t]{2}{*}{ 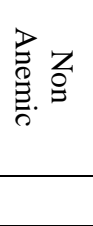 } & \multicolumn{2}{|l|}{ } & \multicolumn{2}{|l|}{ 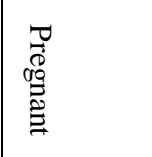 } \\
\hline & & & 1 & 2 & 3 & 4 & 5 & No & $\mathrm{M} \pm \mathrm{SE}$ & & & & & & 1 & 2 & 1 & 2 \\
\hline \multirow[t]{5}{*}{1} & $\leq 20 \mathrm{Y}$ & 31 & 54.8 & 12.9 & 6.5 & 12.9 & 12.9 & 31 & $11.93 \pm 0.28$ & P L & 66.67 & - & & 33.33 & 48.4 & 51.6 & 22.6 & 77.4 \\
\hline & & & & & & & & & & P NL & & & & 100 & & & & \\
\hline & & & & & & & & & & NP L & 25 & 16.67 & & 58.33 & & & & \\
\hline & & & & & & & & & & NP NL & & 37.5 & & 62.5 & & & & \\
\hline & & & & & & & & & & & & & 37.04 & & & & & \\
\hline \multirow[t]{5}{*}{2} & $\leq 30 \mathrm{Y}$ & 249 & 54.2 & 14.5 & 5.2 & 20.5 & 4.0 & 249 & $12.01 \pm 0.0 .8$ & P L & & 50.0 & & 50.0 & 16.9 & 79.5 & 11.6 & 88.4 \\
\hline & & & & & & & & & & P NL & 10.0 & 5.0 & & 85.0 & & & & \\
\hline & & & & & & & & & & NP L & 24.24 & 18.18 & & 57.58 & & & & \\
\hline & & & & & & & & & & NP NL & 5.08 & 44.07 & & 50.85 & & & & \\
\hline & & & & & & & & & & & & & 43.93 & & & & & \\
\hline & & & & & & & & & & & & & & & & & & \\
\hline \multirow[t]{5}{*}{3} & $\leq 40 \mathrm{Y}$ & 307 & 58.3 & 10.1 & 5.2 & 18.9 & 5.2 & 307 & $12.12 \pm 0.08$ & $\mathrm{PL}$ & & & & 100 & 16.9 & 80.5 & 6.5 & 93.5 \\
\hline & & & & & & & & & & P NL & 9.09 & 18.18 & & 72.73 & & & & \\
\hline & & & & & & & & & & NP L & 16.22 & 43.24 & & 40.54 & & & & \\
\hline & & & & & & & & & & NP NL & 7.6 & 37.43 & & 54.97 & & & & \\
\hline & & & & & & & & & & & & & 46.36 & & & & & \\
\hline \multirow{3}{*}{4} & & & & & & & & & & & & & & & & & & \\
\hline & $\leq 50 \mathrm{Y}$ & 52 & 59.61 & 9.62 & 3.85 & 15.38 & 11.54 & 52 & $11.85 \pm 0.17$ & NP NL & 10.0 & 40.0 & & 50.0 & & 100 & & 100 \\
\hline & & & & & & & & & & & & & 50.0 & & & & & \\
\hline & & & & & & & & & & & & & & & & & & \\
\hline \multirow{3}{*}{5} & $\leq 60 \mathrm{Y}$ & 10 & 40.0 & 20.0 & & 10.0 & 30.0 & 10 & $11.30 \pm 0.66$ & NP NL & 50.0 & & & 50.0 & & 100 & & 100 \\
\hline & \multicolumn{18}{|c|}{50.0} \\
\hline & Total & & 56.4 & 12.0 & 5.1 & 18.8 & 6.0 & 649 & & & & & 45.26 & & 16.8 & 80.6 & 8.6 & 91.4 \\
\hline
\end{tabular}

Education: 1: Illiterate; 2: Read and write; 3: Preparatory; 4: High school; 5: University;

Lactating: 1: Lactating; 2: Non Lactating; Pregnant: 1: Pregnant; 2: Non Pregnant;

P L: Pregnant Lactating; P NL: Pregnant Non Lactating; NP L: Non Pregnant Lactating;

NP NL: Non Pregnant Non Lactating; 


\section{Prevalence of Anemia in Egypt (Al-Gharbia Governorate)}

Table (6): Distribution of the sibling according to sex, age, weight, height, BMI, and $\mathrm{Hb}$.

\begin{tabular}{||c|c|c|c|c|c|c|c||}
\hline \hline & & No & Age & Weight $(\mathrm{kg})$ & Height $(\mathrm{cm})$ & $\begin{array}{c}\text { BMI } \\
\mathrm{Kg} / \mathrm{m}^{2}\end{array}$ & $\begin{array}{c}\text { Hb sibling } \\
\mathrm{g} / \mathrm{dl}\end{array}$ \\
\hline \hline \multicolumn{7}{|c|}{ Male } \\
\hline & $6-60$ month & 166 & $23.05 \pm 1.64$ & $11.01 \pm 0.39$ & $80.45 \pm 1.46$ & $16.84 \pm 0.38$ & $11.13 \pm 0.16$ \\
\hline & $5-11$ years & 97 & $8.13 \pm 0.21$ & $23.15 \pm 0.66$ & $121.23 \pm 1.72$ & $15.70 \pm 0.31$ & $11.31 \pm 0.19$ \\
\hline & $11-<13$ years & 20 & $12.03 \pm 0.04$ & $32.57 \pm 1.74$ & $140.43 \pm 1.08$ & $16.52 \pm 0.84$ & $11.47 \pm 0.43$ \\
\hline & $13-14$ years & 28 & $13.47 \pm 0.14$ & $39.00 \pm 1.98$ & $143.00 \pm 3.60$ & $19.35 \pm 1.28$ & $10.55 \pm 0.34$ \\
\hline & $15-18$ years & 38 & $15.63 \pm 0.23$ & $49.08 \pm 3.1$ & $154.45 \pm 3.58$ & $20.3 \pm 0.85$ & $12.40 \pm 0.37$ \\
\hline & $19-30$ years & 6 & $23.67 \pm 2.54$ & $58.58 \pm 3.67$ & $160.00 \pm 1.34$ & $22.96 \pm 1.76$ & $11.55 \pm 1.65$ \\
\hline Total & $0.5: 30$ y & 355 & $6.93 \pm 0.3$ & $22.87 \pm 0.80$ & $109.06 \pm 1.71$ & $17.42 \pm 0.22$ & $11.29 \pm 0.09$ \\
\hline & Female & & & & & \\
\hline & $6-60$ month & 125 & $25.60 \pm 1.60$ & $11.79 \pm 0.38$ & $83.36 \pm 1.46$ & $16.90 \pm 0.28$ & $10.99 \pm 0.19$ \\
\hline & $5-11$ years & 72 & $8.63 \pm 0.20$ & $25.89 \pm 0.83$ & $124.25 \pm 1.35$ & $16.69 \pm 0.44$ & $11.86 \pm 0.18$ \\
\hline & $12-18$ years & 64 & $14.59 \pm 0.21$ & $45.33 \pm 1.64$ & $144.11 \pm 2.18$ & $22.28 \pm 1.08$ & $11.70 \pm 0.19$ \\
\hline & $\geq 19$ years & 6 & $22.00 \pm 0.00$ & $60.00 \pm 0.00$ & $162.00 \pm 0.00$ & $22.86 \pm 0.00$ & $11.90 \pm 0.00$ \\
\hline Total & $0.5: \geq 19$ y & 267 & $7.27 \pm 0.34$ & $24.68 \pm 0.98$ & $110.69 \pm 1.84$ & $18.26 \pm 0.31$ & $11.42 \pm 0.10$ \\
\hline
\end{tabular}

Table (7): Prevalence of anemia in the studied children (sibling)

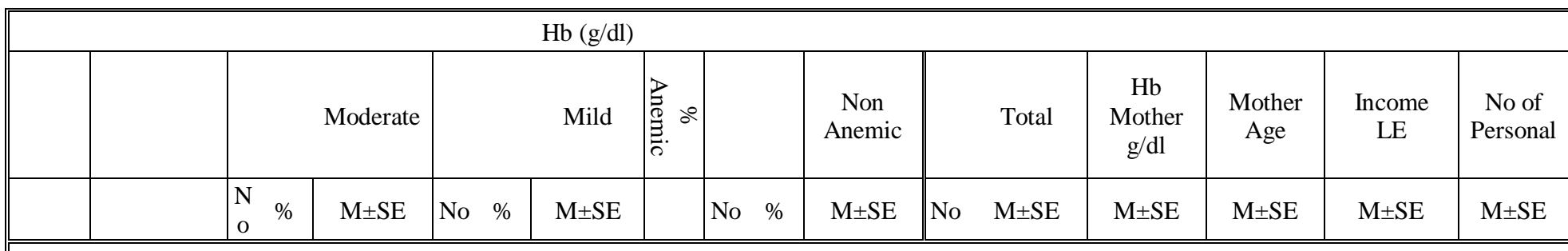

\begin{tabular}{|c|c|c|c|c|c|c|c|c|c|c|c|c|c|c|c|c|c|}
\hline \multirow[t]{8}{*}{ Male } & 6-60 month & 21 & $\begin{array}{c}21.6 \\
5\end{array}$ & $9.12 \pm 0.17$ & 27 & \begin{tabular}{|c|}
27.8 \\
3
\end{tabular} & $\begin{array}{c}10.53 \pm 0.0 \\
5\end{array}$ & 49.48 & 49 & $\begin{array}{c}50.5 \\
2\end{array}$ & $12.32 \pm 0.17$ & 97 & $11.13 \pm 0.16$ & $11.71 \pm 0.16$ & $27.66 \pm 0.57$ & $175.39 \pm 5.60$ & $4.93 \pm 0.13$ \\
\hline & $5-11$ years & $17 \mid$ & $\begin{array}{c}21.7 \\
9\end{array}$ & $8.79 \pm 0.18$ & 22 & $\begin{array}{c}28.2 \\
1\end{array}$ & $\begin{array}{c}10.96 \pm 0.0 \\
9\end{array}$ & 50.0 & 39 & $\begin{array}{c}50.0 \\
0\end{array}$ & $12.59 \pm 0.12$ & 78 & $11.31 \pm 0.19$ & $12.27 \pm 0.14$ & $31.19 \pm 0.43$ & $190.43 \pm 5.25$ & $4.65 \pm 0.13$ \\
\hline & $11-<13$ years & 2 & $\begin{array}{c}14.2 \\
9 \\
\end{array}$ & $8.40 \pm 0.00$ & 6 & $\begin{array}{c}42.8 \\
6 \\
\end{array}$ & \begin{tabular}{|c|}
$11.27 \pm 0.2$ \\
4 \\
\end{tabular} & 57.14 & 6 & $\begin{array}{c}42.8 \\
6 \\
\end{array}$ & $12.70 \pm 0.38$ & 14 & $11.47 \pm 0.43$ & $12.22 \pm 0.47$ & $35.10 \pm 1.01$ & $\begin{array}{c}167.00 \pm 11.7 \\
7\end{array}$ & $4.70 \pm 0.40$ \\
\hline & $13-14$ years & 4 & $\begin{array}{c}33.3 \\
3 \\
\end{array}$ & $9.6 \pm 0.17$ & 6 & $\begin{array}{c}50.0 \\
0 \\
\end{array}$ & \begin{tabular}{|c|}
$10.40 \pm 0.1$ \\
3 \\
\end{tabular} & 83.33 & 2 & $\begin{array}{c}16.6 \\
7 \\
\end{array}$ & $12.90 \pm 0.00$ & 12 & $10.55 \pm 0.34$ & $12.48 \pm 0.3$ & $39.07 \pm 0.97$ & $\begin{array}{c}184.29 \pm 14.9 \\
9\end{array}$ & $4.96 \pm 0.33$ \\
\hline & $15-18$ years & & & - & 9 & $\begin{array}{c}52.9 \\
4 \\
\end{array}$ & \begin{tabular}{|c|}
$11.09 \pm 0.2$ \\
2 \\
\end{tabular} & 52.94 & 8 & $\begin{array}{c}47.0 \\
6 \\
\end{array}$ & $13.88 \pm 0.11$ & 17 & $12.40 \pm 0.37$ & $12.42 \pm 0.45$ & $39.53 \pm 0.81$ & $\begin{array}{c}181.16 \pm 12.1 \\
2\end{array}$ & $4.82 \pm 0.25$ \\
\hline & $19-30$ years & 2 & $\begin{array}{c}50.0 \\
0\end{array}$ & $8.70 \pm 0.00$ & & & - & 50.0 & 2 & $\begin{array}{c}50.0 \\
0\end{array}$ & $14.40 \pm 0.00$ & 4 & $11.55 \pm 1.65$ & $9.50 \pm 0.00$ & $53.0 \pm 0.77$ & $194.0 \pm 29.88$ & $4.33 \pm 0.26$ \\
\hline & & 46 & & & 70 & & & & 106 & & & 222 & $11.29 \pm 0.09$ & $12.04 \pm 0.08$ & $31.64 \pm 0.37$ & $180.66 \pm 2.75$ & $4.82 \pm 0.06$ \\
\hline & & & & & & & & 52.25 & & & & & & & & & \\
\hline
\end{tabular}

\begin{tabular}{|c|c|c|c|c|c|c|c|c|c|c|c|c|c|c|c|c|c|}
\hline \multirow[t]{7}{*}{ Female } & 6-60 month & 28 & $\begin{array}{c}28.5 \\
7 \\
\end{array}$ & $9.09 \pm 0.15$ & 27 & $\begin{array}{c}27.5 \\
5 \\
\end{array}$ & $\begin{array}{c}10.48 \pm 0.0 \\
5 \\
\end{array}$ & 56.12 & 43 & $\begin{array}{c}43.8 \\
8 \\
\end{array}$ & $12.55 \pm 0.24$ & 98 & $10.99 \pm 0.19$ & $11.77 \pm 0.11$ & $28.84 \pm 0.55$ & $182.84 \pm 6.62$ & $4.81 \pm 0.14$ \\
\hline & $5-11$ years & 6 & 9.23 & $8.88 \pm 0.39$ & 23 & $\begin{array}{c}35.3 \\
8 \\
\end{array}$ & $\begin{array}{c}11.01 \pm 0.0 \\
7 \\
\end{array}$ & 44.61 & 36 & $\begin{array}{c}55.3 \\
8 \\
\end{array}$ & $12.91 \pm 0.10$ & 65 & $11.86 \pm 0.18$ & $12.20 \pm 0.15$ & $32.25 \pm 0.62$ & $176.26 \pm 7.45$ & $4.79 \pm 0.15$ \\
\hline & $12-18$ years & 2 & 4.35 & $9.70 \pm 0.00$ & 26 & $\begin{array}{c}56.5 \\
2 \\
\end{array}$ & $\begin{array}{c}10.95 \pm 0.1 \\
0\end{array}$ & 60.87 & 18 & $\begin{array}{c}39.1 \\
3 \\
\end{array}$ & $13.01 \pm 0.19$ & 46 & $11.70 \pm 0.19$ & $12.31 \pm 0.15$ & $38.19 \pm 0.57$ & $174.77 \pm 8.75$ & $5.33 \pm 0.23$ \\
\hline & $\geq 19$ years & & & & 2 & 100 & $\begin{array}{c}11.90 \pm 0.0 \\
0 \\
\end{array}$ & 100.0 & & & - & 2 & $11.90 \pm 0.00$ & $13.10 \pm 0.00$ & $53.00 \pm 0.00$ & $200.00 \pm 0.00$ & $5.00 \pm 0.00$ \\
\hline & & 36 & & & 78 & & & & 97 & & & 211 & $11.42 \pm 0.10$ & $12.04 \pm 0.07$ & & & \\
\hline & & & & & & & & & & & & 433 & & & & & \\
\hline & & & & & & & & 54.03 & & & & & & & & & \\
\hline
\end{tabular}


Ghada Z A Soliman et al

Table (8): Z-Score of height/age; weight/age; weight/height of the studied sibling

\begin{tabular}{|c|c|c|c|c|c|c|c|c|c|}
\hline & \multicolumn{3}{|c|}{ HAZ } & \multicolumn{2}{l|}{ WAZ } & \multicolumn{2}{l|}{ WHZ } \\
\hline & Normal & Tall & Stunted & Normal & $\begin{array}{c}\text { Over } \\
\text { weight }\end{array}$ & $\begin{array}{c}\text { Under } \\
\text { weight }\end{array}$ & Normal & $\begin{array}{c}\text { Over } \\
\text { weight }\end{array}$ & Wasting \\
\hline Male & $\mathbf{5 3 . 0}$ & 10.2 & 36.8 & $\mathbf{7 5 . 2}$ & 4.0 & 20.7 & $\mathbf{7 7 . 1}$ & 11.6 & 11.2 \\
\hline Female & $\mathbf{5 7 . 6}$ & 12.1 & 30.4 & $\mathbf{7 7 . 8}$ & 7.0 & 15.2 & $\mathbf{7 7 . 9}$ & 14.4 & 7.7 \\
\hline
\end{tabular}

\section{Discussion}

Anemia is defined as a low hemoglobin $\mathrm{Hb}$ concentration. Anemia is caused either by limited dietary intake, excessive loss of nutrients or excessive utilization. The main cause of anemia is iron deficiency (ACC/SCN 2000).

The WHO estimates that most preschool children and pregnant women in developing countries and at least 30-40\% in developed countries are iron deficient anemia. The prevalence of anemia in developing countries is three to four times higher than that for developed countries. In developing countries, the most affected population groups are pregnant women (52 $\%$ ) - although all women age 15-59 years are affected $(42 \%)$ - school-age children (48 \%), and preschool children (39\%). Moreover, $45 \%$ of the elderly and $30 \%$ of adult men are anemic, highlighting that the problem extends to other population groups. The problem is more extensive in Southeast Asia and sub-tropical Africa where anemia is linked to poverty.

The incidence of anemia in Egypt (as developing countries) is much higher than developed countries so the present study was aimed to investigate the prevalence of anemia in Al-Gharbia Governorate in Egypt knowing that by the year 2000 the national program of iron supplementation was implemented. The incidence of anemia in infants aged 6-60 moths ranged $49.48-56.12 \%$ for male and female respectively, being higher in female which is different from developed countries as in Norway, where only $2 \%$ to $5 \%$ of infants were found to have IDA (Hay et al. 2004), while other recent reports studied the frequency of IDA in the Arab world found that $7.5 \%$ of Iranian infants aged 6 to 23 months have IDA, while in Jordan a higher frequency of anemia is reported, $72 \%$
(Kilbride et al. 1999 \& 2000) which was higher than reported in this study.

A significant percentage of adolescents in the developing world are anemic, causing considerable health consequences for this age group. About 27 $\%$ of adolescents are estimated to be anemic in developing countries, compared to $6 \%$ in developed countries (deMeyer \& AdielsTegman 1985; and United Nations, Population Division 2000). In this study the data revealed that anemia prevalence in adolescents ranged from 44.61- 62.79\% for male and female respectively, being higher in male and this is comparable with deMeyer \& Adiels-Tegman (1985); United Nations, Population Division (2000) where they suggest that prevalence rates for anemia: In Africa is $45 \%$ for girls and $57 \%$ for boys and also with $\mathrm{SWACH}$ foundation (1997) which found an anemia prevalence rate of $82.9 \%$ among girls in school and $92.7 \%$ among girls not in school (illiterate) in India. On the other hand deMeyer \& Adiels-Tegman (1985); and United Nations, Population Division (2000) found that prevalence of anemia in other countries as Oceania is $45 \%$ for girls and $43 \%$ for boys; in Latin America and the Caribbean is $12 \%$ for girls and $22 \%$ for boys; In Asia is $19 \%$ for girls and $17 \%$ for boys.

In Egypt we found a high rate of anemia. In studies conducted by the International Center for Research on Women, country findings on adolescent anemia among both males and females (Kurz and Johnson-Welch, 1994) where they found high rates of anemia in Nepal (42\%), India (55\%), and Cameroon (32 $\%)$; and they found moderate rates in other

\footnotetext{
${ }^{3}$ SWACH Foundation: Survival for Women and Children Foundation
} 
countries as in Ecuador (17\%) and Jamaica $(16 \%)$.

El-Zanaty and Way, (2001) during $\mathrm{EDHS}^{4}$ revealed that the overall prevalence rate of anemia among women in the reproductive age $15-49$ y was $29.2 \%$ in Egypt. The result of the present study revealed that the overall prevalence of anemia among mothers is 45.26 , which is higher, and also high prevalence rate was recorded among pregnant lactating, nonpregnant lactating, non-pregnant nonlactating mothers $(\geq 47 \%)$ and the least was among pregnant non-lactating (17.14 $\%)$. On the other hand deMeyer \& AdielsTegman (1985) stated that $47 \%$ of women of reproductive age in developing countries are anemic and during pregnancy, $59 \%$ are estimated to be anemic.

The higher prevalence of anemia among the non-pregnant women in this study may be because lactating mother finishing her pregnancy with depleted iron stores, become exposed to the burden of lactation, which is nutritionally demanding. During the lactation period the absence of menstrual blood loss is partially offset by the secretion of $0.3 \mathrm{mg}$ iron/day in breast milk in addition to the basal losses (WHO, 1995 b).

An important finding in the present study is that no severe anemia $(\mathrm{Hb}<7 \mathrm{~g} / \mathrm{dl})$ was found either among mothers or sibling, only moderate to mild anemia was found $((\mathrm{Hb}>7 \mathrm{~g} / \mathrm{dl})$. The majority of anemic mothers have mild anemia (35.13\%).

Causes of anemia among women are multiple and interacting. However one of the important causes may be related to menstrual blood loss, increased duration of menstrual blood flow.

In children anemia compromises physical growth and mental development (Allan and Gillespie, 2001). Previous studies in Egypt indicated that anemia is an important public health problem among children especially among preschool children (El-Sayed et al. 1999). The result of the present study indicates that prevalence of anemia in preschool children and the remaining sibling for both sexes is high ranging $49.48-100 \%$. In preschool children prevalence of anemia reach 49.48

\footnotetext{
${ }^{4}$ EDHS: Egypt demographic and health survey
}

\& 56.12 for male and female respectively being higher in female, which represent a public health problem of high severity. ElZanaty and Way (2001) found that prevalence of anemia was $29.9 \%$ in Egypt, which is different from this result due to different Governorate, different habits, incomes and food habits. The prevalence of anemia in preschool children may be attributed to the negative impact of iron supplementation in antenatal health care facilities because mothers and children don't go there regularly, don't take the pills regularly (because of ignorate or poverty, don't have price of the pills). More than 50 $\%$ of the women (mothers) are illiterate, poor (income $\leq 200 \mathrm{LE}$ ), have bad food habits, bad diet and need more effort to make them understand importance of government program.

The majority of anemic sibling have mild anemia which comprise problem of moderate significant for boys and comprise problem of high significant for girls (table 2) but in general anemia of anemic sibling comprise problem of high significant level of severity (52.25 \& 54.03) for both male and female respectively. Previous studies indicated that anemia is more prevalent among preschool children in the young age (El-Sayed et al. 1999). The prevalence of anemia in this age may be due to their need of iron intake.

It is well known that iron status of pregnant mothers is a key determinant for the young infants's iron store. One of the most common causes of anemia in infants is prematurity. Population that belongs to a lower socioeconomic status has high incidence of IDA. The high incidence of IDA in infants that belong to low-income families is a well-known fact (Kurz and Johnson-Welch 1994, Creed-Kanashiro et al., 1997, and Raina et al., 1997).

\section{Recommendation}

In Egypt although high prevalence of anemia in mothers and their sibling were found but now a days it is slightly lower than before due to government efforts, so prevention of IDA can improve performance in school, life, avoid behavioral alterations, and assure better growth, thus 
allowing children to grow healthier through iron supplementation program. In addition, the burden on the country's economy may be reduced if we prevent IDA at an early age, thus reducing health expenditure in the future. According to the recommendations of the American Academy of Pediatrics (1999), infants who are not breastfed should receive iron-fortified formulas containing 4 to $12 \mathrm{mg} / \mathrm{L}$ of iron, from birth to 12 months of age.

\section{Reference}

1. ACC/SCN (2000): $4 \stackrel{\text { Th }}{\text { Report On The }}$ World Nutrition Situation. ACC/SCN, Geneva, Switzerland.

2. Allan L, And Gilespie S (2001): What Works? A Review Of The Efficacy And Effectiveness Of Nutrition Interventions. ACC/SCN Nutrition Policy Paper No 19.

3. American Academy Of Pediatrics (1999): Committee On Nutrition. Iron Fortification Of Infant Formulas (RE9865). Pediatrics; 104:119-123

4. Avram G (1964): "Quantitative Data." In: Biostatistics: An Introductory Text. Chapter 2. The Macmillan Company, New York, Collier Macmillan Limited, London.

5. CDC (1998): Recommendation To Prevent And Control Iron Deficiency In The United States. MMWR., 47 (No. RR-3).

6. Creed-Kanashiro H, Bentley $M$. Fukumoto M. Et Al. (1997): Relationship Of Anemia To Dietary Intake And Feeding Patterns In Women Of Fertile Age And Adolescent Girls Participating In Community Kitchens In Peri-Urban Lima, Peru." In Improving The Quality Of Iron Supplementation Programs. Mother Care Project/USAID/John Snow, Inc.

7. Demeyer E, Adiels-Tegman M (1985): The Prevalence Of Anemia In The World. World Health Stat Q. 38: 302316.

8. El-Sayed NA, Gad A, Nofal Et Al. (1999): Assesement Of The Prevalence And Potential Determinants Of Nutritional Anæmia In Upper Egypt. Food And Nutrition Bulletin; 20 (4): 417-421.

9. El-Zanaty F, And Way AA (2001): Egypt Demographic And Health Survey (EDHS), 2000. Ministry Of Health And
Population, National Population Council, ORC Marco

10. Florentino RF, And Guirriec RM (1984): Prevalence Of Nutritional Anemia In Infancy And Childhood With Emphasis On Developing Countries. In: Steckel A, Ed. Iron Nutrition In Infancy And Childhood. New York, NY: Raven Press.

11. Hay G, Sansdstad B, Whitelaw A, And Boroch-I B (2004): Iron Status In A Group Of Norwegian Children Aged 6-24 Months. Acta Pediatr; 93:592598.

12. Jelliffe D P, Jelliffee E F P, Zerfas A And Neumann G G (1989): Community Nutritional Assessment. Oxford, New York, Oxford University Press.

13. Joseph M, Michael S, Felice A, Et Al. (2006): The Incidence Of Anemia In An Israeli Population: A Population Analysis For Anemia In 34512 Israeli Infants Aged 9 To 18 Months. Pediatrics; 118; 1055-1060.

14. Karkar PD, And Kotecha PV (2004): Prevalence Of Anemia Among Students Of Nursing School of Vadodara.Nursing J. India, 95: 257-258.

15. Kilbride J, Baker TG, Parapia LA, And Khoury SA. (2000): Incidence Of Iron Deficiency Anemia In Infants In A Prospective Study In Jordan. $\mathrm{Br} \mathrm{J}$ Haematol.; 64:231-236.

16. Kilbride J, Baker TG, Parapia LA, Khoury SA, Shuqaidef SW And Jerwood D (1999): Anaemia During Pregnancy As A Risk Factor For IronDeficiency Anaemia In Infancy: A Case-Control Study In Jordan. Int. J. Epidemiol.; 28: 461-468.

17. Kurz KM, And Johnson-Welch C (1994): The Nutrition And Lives Of Adolescents In Developing Countries: Findings From The Nutrition Of Adolescent Girls Research Program. International Center For Research On Women.

18. Raina N, Gupta A, Sharma M Et Al. (1997): Operational Study On Nutritional Anemia In Pregnant Women, Lactating Women And Adolescent Girls In A Rural Community In India." In Improving The Quality Of Iron Supplementation Programs. Mother Care Project/USAID/ John Snow, Inc.

19. Steel RG, And Torrie JH (1969): "Principles And Procedures Of 
Statistics. Mcgraw Hill, Book Company, Inc., New York.

20. Stolitzfus RJ (2001 A): Defining IronDeficiency Anemia In Public Health Terms: A Tome For Reflection. J Nutr., 131:565S-567S.

21. Stolitzfus RJ (2001 B): IronDeficiency Anemia: Reexamining The Nature And Magnitude Of The Public Health Problem. Summary: Implications For Research And Programs. J. Nutr., 131: 697S-701S.

22. Stolitzfus RJ (2003): Iron Deficiency: Global Prevalence And Consequences. Food Nutr Bull., 24 (4 Suppl): S99S103.

23. SWACH Foundation (1997): Survival For Women And Children (SWACH) Foundation. Anemia In Pregnant Women And Adolescent Girls In Rural Areas Of Haryana, India. Quarterly Progress Report: April To June 1997, Submitted To Mother Care Project, John Snow, Inc.

24. UNAC (United Nations Administrative Committee) (1987): On Coordination, Subcommittee On Nutrition. First Report On The World Nutrition Situation. Rome, Italy: United Nations.

25. United Nations, Population Division, World Population Prospects (2000): The 2000 Revision (Based On Year 1993 Population Estimates).

26. Van Kampen EJ And Zijlstra WG (1961): Standardization Of Hæmoglobincyanide Method. Clin Chem Acta 6: 538.

27. World Bank (1996): Micronutrient Inte-rventions. HDD Flash. Washington D C,
28. WHO Working Group (1986): Use And Interpretation Of Anthropometric Indicators Of Nutritional Status. Bull World Health Org. 64: 929-941.

29. WHO (1985): World Health Organization. Measuring Nutritional Status. Geneva: World Health Organization.

30. WHO (1995 A): World Health Organization. Physical Status: The Use And Interpretation Of Anthropometry. Technical Report Series No.854.Geneva: World Health Organization.

31. WHO (1995 B): World Health Organization. Guidelines For The Control Of Iron Deficiency In Countries Of The Eastern Mediterranean Middle East And North Africa. WHOEM/Nut/177, E/G/11.96.

32. WHO (1996): World Health Organization. Catalogue Of Health Indicators. A Selection Of Important Health Indicators Recommended By WHO Programs. Geneva: World Health Organization; Division Of Health Situation And Trend Assessment.

33. WHO (2001): Iron Deficiency Anaemia: Assessment, Prevention And Control. Geneva: World Health Organization.

34. WHO/UNICEF/UNU (1997): Indicators For Assessing Iron Deficiency And Strategies For Its Prevention. WHO, Geneva.

35. WHO/UNICEF/UNU (2001): Iron Deficiency Anemia: Assessment, Prevention And Control. A Guide For Programme Managers. Available From: Http//Www.Who.Int/Nut/Documents/Id a_Assessment_Prevention_Control. 


\section{انتثار الأنيميا في مصر (محافظة الغربية)}

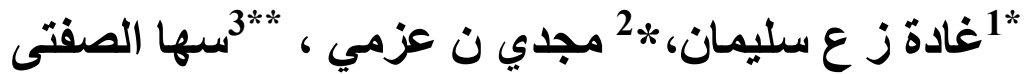

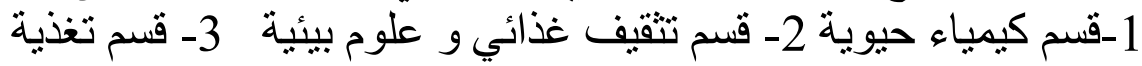

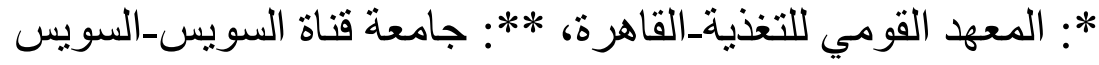

المقدمة:- الأنيميا هي مظهر نقص الحديد و هي النوع الثنائع من نقص التغذية عالميا. و هي

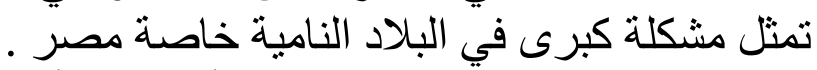

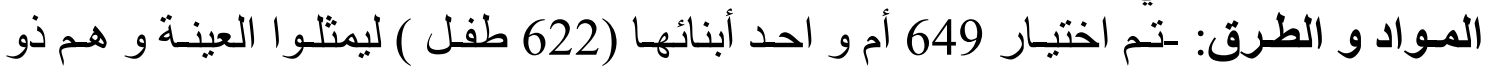

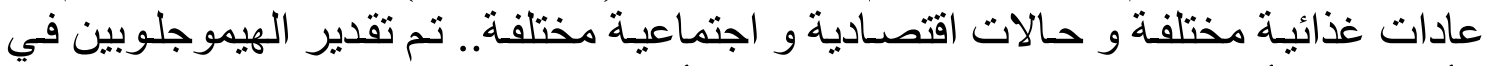

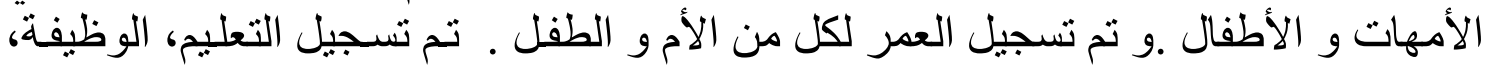

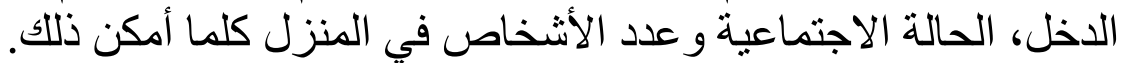

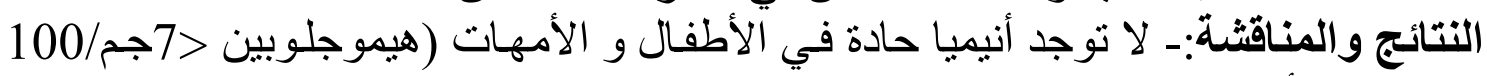

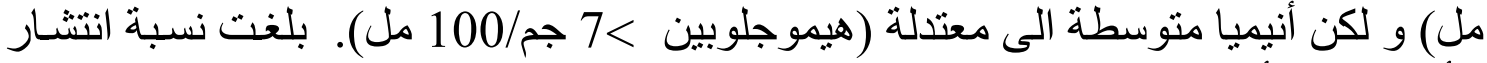

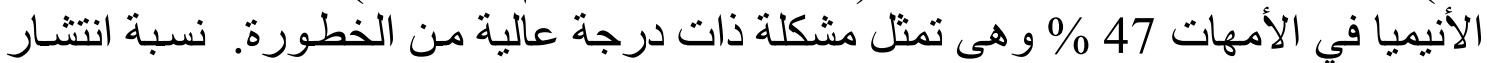

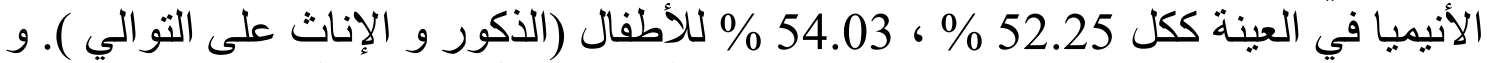

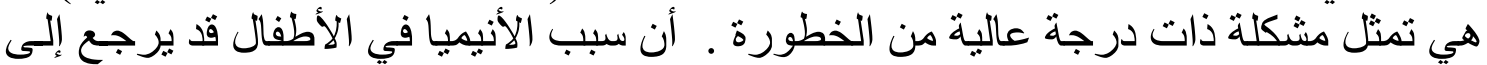

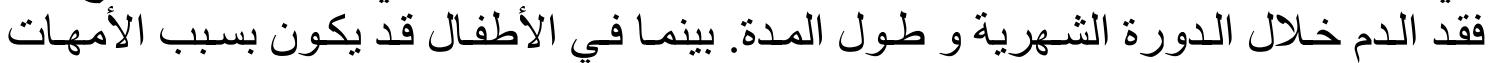

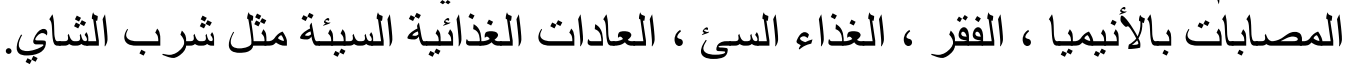
الاستنتاج:- لكي نقلل نسبة انتشار الأنيميا في مصر (الأطفال و الأمهات ) لابد من الندادئ تحسين برنامج تدعيم الحديد. 